\title{
A General and Mild Ullmann-Type Synthesis of Diaryl Ethers
}

\author{
Henri-Jean Cristau,* Pascal P. Cellier, Samy Hamada, Jean-Francis Spindler ${ }^{\S}$ and Marc Taillefer* \\ Laboratoire de Chimie Organique (CNRS UMR 5076) \\ Ecole Nationale Supérieure de Chimie de Montpellier (ENSCM) \\ 8, Rue de l'Ecole Normale \\ 34296 Montpellier Cedex 5, France \\ ${ }^{\S}$ RHODIA Organique Fine, Centre de Recherches de Lyon (CRL) \\ 85, Avenue des Frères Perret, BP 62 \\ 69192 Saint-Fons Cedex, France
}

cristau@cit.enscm.fr and taillefe@ cit.enscm.fr

Supporting Information

\section{General Considerations}

All reactions were carried out in $35 \mathrm{~mL}$ Schlenk tubes under a pure and dry nitrogen atmosphere. Acetonitrile was distilled under nitrogen from $\mathrm{P}_{4} \mathrm{O}_{10}$ and stored on $3 \AA$ activated molecular sieves ${ }^{1}$ under a nitrogen atmosphere. DMF was distilled under vacuum from $\mathrm{MgSO}_{4}$ and stored protected from light on $4 \AA$ activated molecular sieves under a nitrogen atmosphere. All solid materials were stored in the presence of $\mathrm{P}_{4} \mathrm{O}_{10}$ in a benchtop desiccator under vacuum at room temperature and weighed in the air. $\mathrm{K}_{3} \mathrm{PO}_{4}(\mathrm{Fluka}), \mathrm{K}_{2} \mathrm{CO}_{3}$ (Aldrich) and $\mathrm{Cs}_{2} \mathrm{CO}_{3}$ (Aldrich) were ground to a fine powder. Copper (I) iodide and copper (I) bromide were purified according to literature procedures. ${ }^{2}$ The former was stored protected from light. Copper (I) oxide and copper (II) oxide (both Aldrich) were used without further purification. Salicylaldoxime and dimethylglyoxime were purchased from commercial sources. The former was recrystallized in petroleum ether prior to use. All aryl halides and phenols were purchased from commercial sources (Aldrich, Acros, Avocado, Fluka, Lancaster). If solids, they were recrystallized in an appropriate solvent. ${ }^{3}$ If liquids, they were distilled under vacuum and stored under an atmosphere of nitrogen. Special care was taken with liquid aryl iodides: the samples were regularly distilled over copper powder to remove iodine and stored protected from light. All phenols were also stored protected from light. Molecular sieves were activated and stored under vacuum at $100{ }^{\circ} \mathrm{C}$ in the presence of $\mathrm{P}_{4} \mathrm{O}_{10}$.

Isolated yields refer to compounds estimated to be $95 \%$ pure or higher as determined by GC and ${ }^{1} \mathrm{H}$ NMR. Flash column chromatography was performed with SDS 60 A C.C silica gel (35-70 $\mu \mathrm{m}$ or 70-200 $\mu \mathrm{m})$. Thin layer chromatography was carried out using Merck silica gel $60 \mathrm{~F}_{254}$ plates. All products were characterized by ${ }^{1} \mathrm{H}$ NMR, ${ }^{13} \mathrm{C}$ NMR and GC/MS. New compounds and previously partially characterized compounds were further characterized by IR and elemental analysis. IR spectra were recorded on a Nicolet 210 FT-IR instrument (neat, thin film for liquid products and $\mathrm{KBr}$ pellet or in dichloromethane solution for solid products). Elemental analysis was performed by the CNRS Service Central d'Analyse, Vernaison, France. ${ }^{1} \mathrm{H}$ NMR and ${ }^{13} \mathrm{C}\left\{{ }^{1} \mathrm{H}\right\}$ NMR spectra were recorded at room temperature on a Bruker AC $200 \mathrm{MHz}$ or a Bruker Avance $250 \mathrm{MHz}$ 
instrument with chemical shifts reported in ppm relative to the residual deuterated solvent peak. ${ }^{19} \mathrm{~F}\left\{{ }^{1} \mathrm{H}\right\} \mathrm{NMR}$ spectra were recorded at room temperature on a Bruker Avance $250 \mathrm{MHz}$ instrument with chemical shifts reported in $\mathrm{ppm}$ relative to $\mathrm{CFCl}_{3}$. The peak patterns are indicated as s, singlet; $\mathrm{d}$, doublet; t, triplet; $\mathrm{q}$, quadruplet; dd, doublet of doublets; m, multiplet. Gas chromatographic analysis were performed on a Delsi Nermag DI-200 instrument with a FID detector, a Delsi Nermag Enica 31 integrator and a SGE BPX5 25 m x $0.53 \mathrm{~mm}$ semi-capillary apolar column (Stationary phase: $5 \%$ phenylpolysil-phenylenesiloxane film, $1 \mu \mathrm{m}$ ). Gas chromatography - mass spectra (GC/MS) were recorded on an Agilent Technologies $6890 \mathrm{~N}$ instrument with an Agilent $5973 \mathrm{~N}$ mass detector (EI) and a HP5-MS $30 \mathrm{~m}$ x $0.25 \mathrm{~mm}$ capillary apolar column (Stationary phase: 5 $\%$ diphenyldimethylpolysiloxane film, $0.25 \mu \mathrm{m})$. FAB+ mass spectra were recorded on a JEOL JMS-DX300 spectrometer (3 Kev, xenon) in a $m$-nitrobenzylalcohol matrix. Melting points were determined using a Büchi B540 apparatus and are uncorrected.

\section{General procedure for the O-arylation of phenols ( 2 mmol scale)}

After standard cycles of evacuation and back-filling with dry and pure nitrogen, an oven-dried Schlenk tube equipped with a magnetic stirring bar was charged with $\mathrm{Cu}_{2} \mathrm{O}(0.1 \mathrm{mmol})$, the ligand $(0.4 \mathrm{mmol}), \mathrm{Cs}_{2} \mathrm{CO}_{3}$ (4.0 mmol), activated and powdered $3 \AA$ molecular sieves $(600 \mathrm{mg})$, the phenol coupling partner $(2.0 \mathrm{mmol})$, if a solid, and the aryl halide $(3.0 \mathrm{mmol})$, if a solid. The tube was evacuated, back-filled with nitrogen and capped with a rubber septum. If liquids, the phenol and the aryl halide were added under a stream of nitrogen by syringe at room temperature, followed by anhydrous and degassed acetonitrile or DMF (1.2 mL). The septum was removed, the tube sealed under a positive pressure of nitrogen and stirred in an oil bath (preheated to $82{ }^{\circ} \mathrm{C}$ or $110{ }^{\circ} \mathrm{C}$ ), for the required time period. The reaction mixture was allowed to cool to room temperature, diluted with dichloromethane and filtered through a plug of celite ${ }^{\circledR}$, the filter cake being further washed with dichloromethane $(\sim 20 \mathrm{~mL})$. The filtrate was concentrated in vacuo to yield the crude product, that was purified by silica gel chromatography with an eluent of hexanes and dichloromethane.

\section{General procedure for reactivity comparisons of phenols or aryl halides (0.5 mmol scale)}

After standard cycles of evacuation and back-filling with dry and pure nitrogen, a $35 \mathrm{~mL}$ oven-dried Schlenk tube equipped with a magnetic stirring bar $(12 \times 4.5 \mathrm{~mm})$ was charged with $\mathrm{Cu}_{2} \mathrm{O}(25 \mu \mathrm{mol})$, the ligand (0.1 mmol), $\mathrm{Cs}_{2} \mathrm{CO}_{3}(1.0 \mathrm{mmol})$, activated and powdered $3 \AA$ molecular sieves $(150 \mathrm{mg})$, the phenol coupling partner $(0.5 \mathrm{mmol})$, if a solid, and the aryl halide $(0.75 \mathrm{mmol})$, if a solid. The tube was evacuated, back-filled with nitrogen and capped with a rubber septum. If liquids, the phenol and the aryl halide were added under a stream of nitrogen by syringe at room temperature, followed by anhydrous and degassed acetonitrile or DMF $(300 \mu \mathrm{L})$. The septum was removed, the tube sealed under a positive pressure of nitrogen and stirred in an oil bath (preheated to $82{ }^{\circ} \mathrm{C}$ or $110^{\circ} \mathrm{C}$ ), for the required time period. The reaction mixture was allowed to cool to room temperature and was diluted with dichloromethane $(5 \mathrm{~mL}) .65 \mu \mathrm{L}$ of 1,3-dimethoxybenzene (internal standard) were added. A small sample of the reaction mixture was taken and filtered through a plug of celite ${ }^{\circledR}$, the filter cake being further washed with dichloromethane. The filtrate was washed three times with water and analyzed by gas chromatography. The GC yields were determined by obtaining the correction factors using authentic samples of the expected products. 


\section{Experimental procedures and characterization data}

\section{Synthesis of trans-1,2-bis(2'-pyridylidenamino)-cyclohexane (Chxn-Py-Al, 1)}

\section{Experimental procedure}

To a solution of 2-pyridylaldehyde $(6.66 \mathrm{~mL}, 70.0 \mathrm{mmol})$ in absolute ethanol $(50 \mathrm{~mL})$ were successively added anhydrous magnesium sulphate $(12.65 \mathrm{~g}, 105.1 \mathrm{mmol})$ and rac-trans-1,2-diaminocyclohexane $(4.2 \mathrm{~mL}, 35.0 \mathrm{mmol})$. The mixture was stirred for 20 hours at room temperature, heated at reflux for 2.5 hours and filtered through a frit while still hot. The solid was discarded and the filtrate was concentrated in vасио. The residue was recrystallized in ethanol to provide $8.2 \mathrm{~g}(80 \%$ yield) of the desired product as pale yellow crystals. Only the $1 \mathrm{~S}, 2 \mathrm{~S}$ stereoisomer is known in literature. ${ }^{4}$

\section{$\underline{\text { Identification }}$}

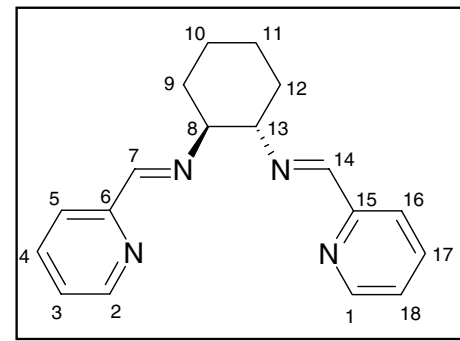

Mp: $140-141^{\circ} \mathrm{C}(\mathrm{EtOH})$.

${ }^{1} \mathbf{H}$ NMR (250 MHz, $\left.\mathbf{C D C l}_{3}\right): \delta 8.54\left(\mathrm{ddd}, 2 \mathrm{H},{ }^{3} \mathrm{~J}_{\mathrm{HH}}=4.9 \mathrm{~Hz},{ }^{4} \mathrm{~J}_{\mathrm{HH}}=1.7 \mathrm{~Hz}\right.$, $\left.{ }^{5} \mathrm{~J}_{\mathrm{HH}}=1.0 \mathrm{~Hz}, \mathrm{H}_{1,2}\right), 8.30\left(\mathrm{~s}, 2 \mathrm{H}, \mathrm{H}_{7,14}\right), 7.87\left(\mathrm{ddd}, 2 \mathrm{H},{ }^{3} \mathrm{~J}_{\mathrm{HH}}=7.9 \mathrm{~Hz},{ }^{4} \mathrm{~J}_{\mathrm{HH}}=\right.$ $\left.1.5 \mathrm{~Hz},{ }^{5} \mathrm{~J}_{\mathrm{HH}}=1.0 \mathrm{~Hz}, \mathrm{H}_{5,16}\right), 7.63$ (dddd, $2 \mathrm{H},{ }^{3} \mathrm{~J}_{\mathrm{HH}}=7.9 \mathrm{~Hz},{ }^{3} \mathrm{~J}_{\mathrm{HH}}=7.5 \mathrm{~Hz}$, $\left.{ }^{4} \mathrm{~J}_{\mathrm{HH}}=1.7 \mathrm{~Hz},{ }^{5} \mathrm{~J}_{\mathrm{HH}}=0.6 \mathrm{~Hz}, \mathrm{H}_{4,17}\right), 7.22\left(\mathrm{ddd}, 2 \mathrm{H},{ }^{3} \mathrm{~J}_{\mathrm{HH}}=7.5 \mathrm{~Hz},{ }^{3} \mathrm{~J}_{\mathrm{HH}}=4.9\right.$ $\left.\mathrm{Hz},{ }^{4} \mathrm{~J}_{\mathrm{HH}}=1.5 \mathrm{~Hz}, \mathrm{H}_{3,18}\right), 3.50\left(\mathrm{~m}, 2 \mathrm{H}, \mathrm{H}_{8,13}\right), 1.83(\mathrm{~m}, 6 \mathrm{H}), 1.40-1.55(\mathrm{~m}$, $2 \mathrm{H})$.

${ }^{13} \mathbf{C}$ NMR $\left\{{ }^{1} \mathbf{H}\right\}\left(\mathbf{5 0} \mathbf{~ M H z}, \mathbf{C D C l}_{3}\right): \delta 161.42\left(\mathrm{C}_{7,14}\right), 154.61\left(\mathrm{C}_{6,15}\right), 149.21$ $\left(\mathrm{C}_{1,2}\right), 136.39\left(\mathrm{C}_{4,17}\right), 124.43\left(\mathrm{C}_{3,18}\right), 121.29\left(\mathrm{C}_{5,16}\right), 73.53\left(\mathrm{C}_{8,13}\right), 32.70$ $\left(\mathrm{C}_{9,12}\right), 24.33\left(\mathrm{C}_{10,11}\right)$.

FAB+ (NBA matrix) : M/Z $293(100 \%, \mathrm{M}+1), 107$ (52\%), $92(38 \%), 119$ (25\%), 294 (23\%, M+2), 204 (22\%), 79 (21\%), 187 (20\%), 585 (1\%, $2 \mathrm{M}+1)$

IR (KBr) : $v\left(\mathrm{~cm}^{-1}\right)=3273,3071,3055,3050,2941,2934,2925,2865$, 2857, 2850, 1644, 1586, 1566, 1467, 1449, 1433, 1372, 1338, 991, 934, 867, $839,771,743$.

\section{Copper-Catalyzed Synthesis of Diaryl Ethers}

\section{1) 2-methyl-diphenylether ${ }^{5}$}

\section{Experimental procedure (Table 5, entry 5)}

Following the general procedure $\left(82{ }^{\circ} \mathrm{C}, 40\right.$ hours $), o$-cresol $(206 \mu \mathrm{L}, 2.0 \mathrm{mmol})$ was coupled with iodobenzene $(336 \mu \mathrm{L}, 3.0 \mathrm{mmol})$ using cesium carbonate $(1.303 \mathrm{~g}, 4.0 \mathrm{mmol}), \mathrm{Cu}_{2} \mathrm{O}(14.4 \mathrm{mg}, 0.1 \mathrm{mmol})$, Chxn-Py-Al (117 mg, $0.4 \mathrm{mmol})$, activated and powdered $3 \AA$ molecular sieves (600 mg), and acetonitrile (1.2 $\mathrm{mL}$ ). The crude oily residue was purified by flash chromatography on silica gel (eluent: hexanes) to provide 343 $\mathrm{mg}\left(93 \%\right.$ yield) of the desired product as a colorless oil, which can be crystallized in a few hours if left at $0{ }^{\circ} \mathrm{C}$ (colorless crystals, Lit. ${ }^{5}: \mathrm{Mp}=21.5-22{ }^{\circ} \mathrm{C}$ ).

\section{$\underline{\text { Identification }}$}

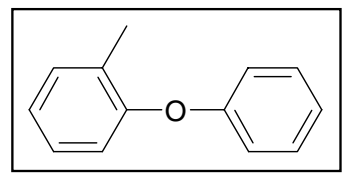

${ }^{1} \mathbf{H}$ NMR (200 MHz, $\left.\mathbf{C D C l}_{3}\right): \delta$ 7.19-7.35 (m, 3H), 7.00-7.18 (m, 3H), 6.87$6.94(\mathrm{~m}, 3 \mathrm{H}), 2.25\left(\mathrm{~s}, 3 \mathrm{H}, \mathrm{CH}_{3}\right)$.

${ }^{13} \mathbf{C}$ NMR $\left\{{ }^{1} \mathbf{H}\right\}\left(\mathbf{5 0} \mathbf{~ M H z}, \mathbf{C D C l}_{3}\right): \delta 158.08(\mathrm{Cq}), 154.60(\mathrm{Cq}), 131.60$ $(\mathrm{CH}), 130.14(\mathrm{Cq}), 129.81(2 \mathrm{CH}), 127.30(\mathrm{CH}), 124.15(\mathrm{CH}), 122.48(\mathrm{CH})$, $119.94(\mathrm{CH}), 117.44(2 \mathrm{CH}), 16.35\left(\mathrm{CH}_{3}\right)$.

GC/MS (EI): $\mathrm{rt}=15.25 \mathrm{~min}, \mathrm{M} / \mathrm{Z}=184$.

$\mathbf{R}_{\mathbf{f}}: 0.36$ (eluent: hexanes). 


\section{2) Diphenylether}

\section{Experimental procedure (Table 5, entry 6)}

Following the general procedure $\left(82{ }^{\circ} \mathrm{C}, 24\right.$ hours $)$, phenol $(188 \mathrm{mg}, 2.0 \mathrm{mmol})$ was coupled with iodobenzene $(336 \mu \mathrm{L}, 3.0 \mathrm{mmol})$ using cesium carbonate $(1.303 \mathrm{~g}, 4.0 \mathrm{mmol}), \mathrm{Cu}_{2} \mathrm{O}(14.4 \mathrm{mg}, 0.1 \mathrm{mmol})$, salicylaldoxime ( $55 \mathrm{mg}, 0.4 \mathrm{mmol})$, activated and powdered $3 \AA$ A molecular sieves $(600 \mathrm{mg})$, and acetonitrile $(1.2$ $\mathrm{mL}$ ). The crude oily residue was purified by flash chromatography on silica gel (eluent: hexanes) to provide 344 $\mathrm{mg}$ (quantitative yield) of the desired product as a colorless oil, which can be crystallized in a few hours if left at $-5{ }^{\circ} \mathrm{C}$ (colorless crystals).

\section{$\underline{\text { Identification }}$}

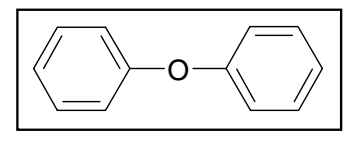

Mp: $26^{\circ} \mathrm{C}\left(\right.$ Lit. $\left.^{6}: 26.85^{\circ} \mathrm{C}\right)$.

${ }^{1}$ H NMR (200 MHz, $\left.\mathbf{C D C l}_{3}\right): \delta$ 7.37-7.47 (m, 4H), 7.10-7.23 (m, 6H).

${ }^{13} \mathbf{C}$ NMR $\left\{{ }^{1} \mathbf{H}\right\}\left(\mathbf{5 0} \mathbf{~ M H z}, \mathbf{C D C l}_{3}\right): \delta 157.38(2 \mathrm{Cq}), 129.88(4 \mathrm{CH}), 123.35$

$(2 \mathrm{CH}), 119.02(4 \mathrm{CH})$.

GC/MS (EI): $\mathrm{rt}=14.43 \mathrm{~min}, \mathrm{M} / \mathrm{Z}=170$.

$\mathbf{R}_{\mathbf{f}}: 0.33$ (eluent: hexanes).

\section{3) 4-t-butyl-diphenylether}

\section{Experimental procedure (Table 5, entry 7)}

Following the general procedure $\left(82^{\circ} \mathrm{C}, 25\right.$ hours), 4-t-butylphenol (300 $\mathrm{mg}, 2.0 \mathrm{mmol}$ ) was coupled with iodobenzene $(336 \mu \mathrm{L}, 3.0 \mathrm{mmol})$ using cesium carbonate $(1.303 \mathrm{~g}, 4.0 \mathrm{mmol}), \mathrm{Cu}_{2} \mathrm{O}(14.4 \mathrm{mg}, 0.1 \mathrm{mmol})$, Chxn-Py-Al (117 mg, $0.4 \mathrm{mmol})$, activated and powdered $3 \AA$ molecular sieves $(600 \mathrm{mg})$, and acetonitrile $(1.2$ $\mathrm{mL}$ ). The crude oily residue was purified by flash chromatography on silica gel (eluent: hexanes) to provide 430 $\mathrm{mg}\left(95 \%\right.$ yield) of the desired product as a colorless oil, which can be crystallized in a few hours if left at $-5{ }^{\circ} \mathrm{C}$ (colorless crystals).

\section{$\underline{\text { Identification }}$}

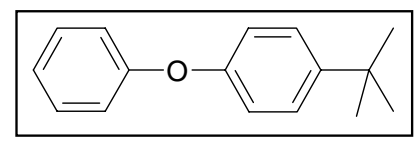

Mp: $52{ }^{\circ} \mathrm{C}\left(\right.$ Lit. $\left.^{7}: 53-54{ }^{\circ} \mathrm{C}\right)$.

${ }^{1}$ H NMR (200 MHz, DMSO- $\left.d_{6}\right): \delta$ 7.33-7.41 (m, 4H), 7.06-7.14 (m, $\left.1 \mathrm{H}\right)$, 6.91-6.99 (m, 4H), 1.27 (s, 9H, Me).

${ }^{13} \mathbf{C}$ NMR $\left\{{ }^{1} \mathbf{H}\right\}$ (50 MHz, DMSO-d $\left.\boldsymbol{d}_{\mathbf{6}}\right): \delta 156.94(\mathrm{Cq}), 154.09(\mathrm{Cq}), 145.73$

$(\mathrm{Cq}), 129.88(2 \mathrm{CH}), 126.61(2 \mathrm{CH}), 123.05(\mathrm{CH}), 118.21(4 \mathrm{CH}), 33.96$ $(\mathrm{Cq}), 31.18\left(3 \mathrm{CH}_{3}\right)$.

GC/MS (EI): $\mathrm{rt}=18.50 \mathrm{~min}, \mathrm{M} / \mathrm{Z}=226$.

$\mathbf{R}_{\mathbf{f}}: 0.36$ (eluent: hexanes).

\section{4) 4-methoxy-diphenylether ${ }^{8}$}

\section{Experimental procedure (Table 5, entry 8)}

Following the general procedure $\left(82{ }^{\circ} \mathrm{C}, 28\right.$ hours $)$, 4-hydroxyanisole $(248 \mathrm{mg}, 2.0 \mathrm{mmol})$ was coupled with iodobenzene $(336 \mu \mathrm{L}, 3.0 \mathrm{mmol})$ using cesium carbonate $(1.303 \mathrm{~g}, 4.0 \mathrm{mmol}), \mathrm{Cu}_{2} \mathrm{O}(14.4 \mathrm{mg}, 0.1 \mathrm{mmol})$, Chxn-Py-Al (117 mg, $0.4 \mathrm{mmol})$, activated and powdered $3 \AA$ molecular sieves $(600 \mathrm{mg})$, and acetonitrile (1.2 $\mathrm{mL}$ ). The crude orange oil was purified by flash chromatography on silica gel (eluent: hexanes / dichloromethane $100 / 0$ to $95 / 5$ ) to provide $380 \mathrm{mg}$ ( $95 \%$ yield) of the desired product as a colorless oil. 


\section{$\underline{\text { Identification }}$}

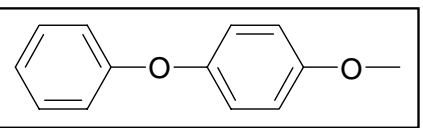

${ }^{1} \mathbf{H}$ NMR (200 MHz, CDCl $)$ ): $\delta$ 7.30-7.39 (m, 2H), 6.89-7.09 (m, 7H), 3.84 $(\mathrm{s}, 3 \mathrm{H})$.

${ }^{13} \mathbf{C}$ NMR $\left\{{ }^{1} \mathbf{H}\right\}\left(\mathbf{5 0} \mathbf{~ M H z}, \mathbf{C D C l}_{3}\right): \delta 158.60(\mathrm{Cq}), 155.97(\mathrm{Cq}), 150.18(\mathrm{Cq})$, $129.69(2 \mathrm{CH}), 122.49(\mathrm{CH}), 120.91(2 \mathrm{CH}), 117.64(2 \mathrm{CH}), 114.92(2 \mathrm{CH})$, $55.67\left(\mathrm{CH}_{3}\right)$.

GC/MS (EI): $\mathrm{rt}=17.67 \mathrm{~min}, \mathrm{M} / \mathrm{Z}=200$.

$\mathbf{R}_{\mathbf{f}}$ : 0.25 (eluent: hexanes / dichloromethane 80/20).

5) 2,4-dichloro-diphenylether ${ }^{9}$

Experimental procedure (Table 5, entry 9)

Following the general procedure $\left(82{ }^{\circ} \mathrm{C}, 40\right.$ hours), 2,4-dichlorophenol (326 $\left.\mathrm{mg}, 2.0 \mathrm{mmol}\right)$ was coupled with iodobenzene $(336 \mu \mathrm{L}, 3.0 \mathrm{mmol})$ using cesium carbonate $(1.303 \mathrm{~g}, 4.0 \mathrm{mmol}), \mathrm{Cu}_{2} \mathrm{O}(14.4 \mathrm{mg}, 0.1$ mmol), salicylaldoxime $(55 \mathrm{mg}, 0.4 \mathrm{mmol})$, activated and powdered $3 \AA$ molecular sieves $(600 \mathrm{mg})$, and acetonitrile $(1.2 \mathrm{~mL})$. The crude product was purified by flash chromatography on silica gel (eluent: hexanes) to provide $191 \mathrm{mg}$ (40\% yield) of the desired product as a colorless oil.

\section{$\underline{\text { Identification }}$}

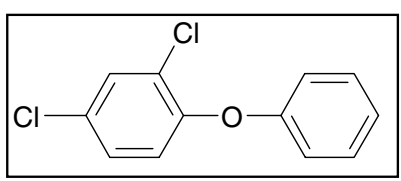

${ }^{1} \mathbf{H}$ NMR (200 MHz, CDCl $): \delta 7.50\left(\mathrm{dd}, 1 \mathrm{H},{ }^{4} \mathrm{~J}_{\mathrm{HH}}=2.3 \mathrm{~Hz},{ }^{5} \mathrm{~J}_{\mathrm{HH}}=0.6 \mathrm{~Hz}\right.$, $\left.\mathrm{H}_{4}\right), 7.34-7.42(\mathrm{~m}, 2 \mathrm{H}), 7.15-7.19(\mathrm{~m}, 2 \mathrm{H}), 6.90-7.02(\mathrm{~m}, 3 \mathrm{H})$.

${ }^{13} \mathbf{C}$ NMR $\left\{{ }^{1} \mathbf{H}\right\}\left(\mathbf{5 0} \mathbf{~ M H z}, \mathbf{C D C l}_{3}\right): \delta 156.66(\mathrm{Cq}), 151.49(\mathrm{Cq}), 130.52$ $(\mathrm{CH}), 129.96(2 \mathrm{CH}), 129.16(\mathrm{Cq}), 128.08(\mathrm{CH}), 126.65(\mathrm{Cq}), 123.78(\mathrm{CH})$, $121.39(\mathrm{CH}), 118.07(2 \mathrm{CH})$.

GC/MS (EI): $\mathrm{rt}=18.51 \mathrm{~min}, \mathrm{M} / \mathrm{Z}=238,240$ and 242 .

$\mathbf{R}_{\mathbf{f}}: 0.40$ (eluent: hexanes).

6) 3,5-dimethyl-diphenylether

Experimental procedure (Table 5, entry 11)

Following the general procedure $\left(82{ }^{\circ} \mathrm{C}, 29\right.$ hours $), 3,5$-dimethylphenol $(244 \mathrm{mg}, 2.0 \mathrm{mmol})$ was coupled with iodobenzene $(336 \mu \mathrm{L}, 3.0 \mathrm{mmol})$ using cesium carbonate $(1.303 \mathrm{~g}, 4.0 \mathrm{mmol}), \mathrm{Cu}_{2} \mathrm{O}(14.4 \mathrm{mg}, 0.1$ mmol), Chxn-Py-Al (117 mg, $0.4 \mathrm{mmol})$, activated and powdered $3 \AA$ molecular sieves (600 mg), and acetonitrile $(1.2 \mathrm{~mL})$. The crude brown oil was purified by flash chromatography on silica gel (eluent: hexanes) to provide $381 \mathrm{mg}$ (96\% yield) of the desired product as a colorless oil.

\section{$\underline{\text { Identification }}$}

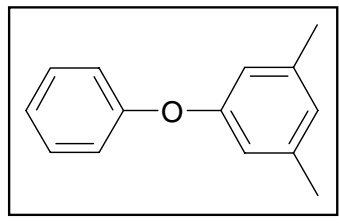

${ }^{1} \mathbf{H}$ NMR (200 MHz, $\left.\mathbf{C D C l}_{3}\right): \delta^{10} 7.28-7.42(\mathrm{~m}, 2 \mathrm{H}), 7.12-7.17(\mathrm{~m}, 1 \mathrm{H})$, 7.03-7.14 (m, 2H), $6.79(\mathrm{~m}, 1 \mathrm{H}), 6.69(\mathrm{~m}, 2 \mathrm{H}), 2.33\left(\mathrm{~s}, 6 \mathrm{H}, \mathrm{CH}_{3}\right)$.

${ }^{13} \mathbf{C}$ NMR $\left\{{ }^{1} \mathbf{H}\right\}\left(\mathbf{5 0} \mathbf{~ M H z}, \mathbf{C D C l}_{3}\right): \delta^{10} 157.50(\mathrm{Cq}), 157.22(\mathrm{Cq}), 139.61(2$ $\mathrm{Cq}), 129.70(2 \mathrm{CH}), 125.04(\mathrm{CH}), 123.02(\mathrm{CH}), 118.89(2 \mathrm{CH}), 116.67$ (2 $\mathrm{CH}), 21.35\left(2 \mathrm{CH}_{3}\right)$.

GC/MS (EI): $\mathrm{rt}=16.87 \mathrm{~min}, \mathrm{M} / \mathrm{Z}=198$.

$\mathbf{R}_{\mathbf{f}}: 0.19$ (eluent: hexanes). 
7) 3',5'-dimethyl-4-cyano-diphenylether ${ }^{11}$

Experimental procedure (Table 5, entry 12)

Following the general procedure $\left(82{ }^{\circ} \mathrm{C}, 24\right.$ hours), 3,5-dimethylphenol $(244 \mathrm{mg}, 2.0 \mathrm{mmol})$ was coupled with 4-iodobenzonitrile $(595 \mathrm{mg}, 2.6 \mathrm{mmol})$ using cesium carbonate $(1.303 \mathrm{~g}, 4.0 \mathrm{mmol}), \mathrm{Cu}_{2} \mathrm{O}(14.4$ mg, $0.1 \mathrm{mmol})$, Chxn-Py-Al (117 mg, $0.4 \mathrm{mmol})$, activated and powdered $3 \AA$ molecular sieves $(600 \mathrm{mg})$, and acetonitrile $(1.6 \mathrm{~mL})$. The crude product was dried for 2 hours at $100{ }^{\circ} \mathrm{C}$ and then purified by flash chromatography on silica gel (eluent: gradient hexanes / dichloromethane 100/0 to 50/50) to provide $415 \mathrm{mg}$ (93 $\%$ yield) of the desired product as an orange oil.

\section{$\underline{\text { Identification }}$}

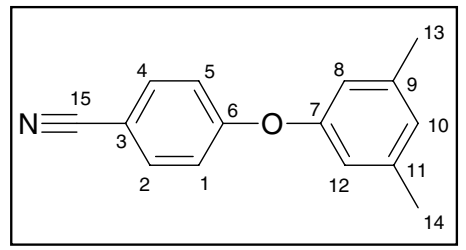

Mp: $58^{\circ} \mathrm{C}$.

${ }^{1}$ H NMR (200 MHz, $\left.\mathbf{C D C l}_{3}\right)$ : $\delta$ 7.53-7.60 (m, 2H), 6.95-7.00 (m, 2H), $6.86(\mathrm{~m}, 1 \mathrm{H}), 6.68(\mathrm{~m}, 2 \mathrm{H}), 2.32\left(\mathrm{~s}, 6 \mathrm{H}, \mathrm{CH}_{3}\right)$.

${ }^{13} \mathbf{C} \mathbf{N M R}\left\{{ }^{1} \mathbf{H}\right\}\left(\mathbf{5 0} \mathbf{M H z}, \mathbf{C D C l}_{3}\right): \delta 161.90\left(\mathrm{C}_{6}\right), 154.76\left(\mathrm{C}_{7}\right), 140.17$ $\left(\mathrm{C}_{9,11}\right), 134.07\left(\mathrm{C}_{2,4}\right), 126.86\left(\mathrm{C}_{10}\right), 118.92\left(\mathrm{C}_{15}\right), 118.03(2 \mathrm{CH}), 117.88(2$ $\mathrm{CH}), 105.55\left(\mathrm{C}_{3}\right), 21.28\left(\mathrm{C}_{13,14}\right)$.

GC/MS (EI): $\mathrm{rt}=20.54 \mathrm{~min}, \mathrm{M} / \mathrm{Z}=223$.

IR $($ KBr $): v\left(\mathrm{~cm}^{-1}\right)=3058,3017,2958,2230(\mathrm{CN}), 1603,1587,1504$, 1298, 1239 (C-O), 1166, 1134, 1024, 950, 859, 838.

$\mathbf{R}_{\mathbf{f}}: 0.32$ (eluent: hexanes / dichloromethane 50/50).

8) 3',5'-dimethyl-4-trifluoromethyl-diphenylether

Experimental procedure (Table 5, entry 13)

Following the general procedure $\left(82{ }^{\circ} \mathrm{C}, 24\right.$ hours $), 3,5$-dimethylphenol $(244 \mathrm{mg}, 2.0 \mathrm{mmol})$ was coupled with 1-iodo-4-trifluoromethyl-benzene ( $294 \mu \mathrm{L}, 2.6 \mathrm{mmol})$ using cesium carbonate $(1.303 \mathrm{~g}, 4.0 \mathrm{mmol})$, $\mathrm{Cu}_{2} \mathrm{O}$ (14.4 mg, $\left.0.1 \mathrm{mmol}\right)$, Chxn-Py- $\mathrm{Al}(117 \mathrm{mg}, 0.4 \mathrm{mmol})$, activated and powdered $3 \AA$ A molecular sieves (600 $\mathrm{mg}$ ), and acetonitrile $(1.2 \mathrm{~mL})$. The crude product was purified by flash chromatography on silica gel (eluent: hexanes) to provide $506 \mathrm{mg}$ ( $95 \%$ yield) of the desired product as an orange oil.

\section{$\underline{\text { Identification }}$}

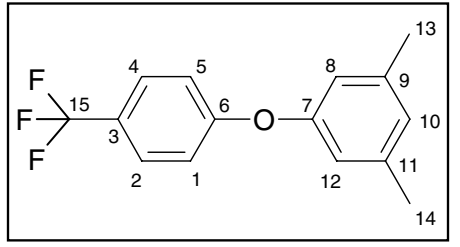

${ }^{1}$ H NMR $\left(200 \mathrm{MHz}, \mathbf{C D C l}_{3}\right): \delta 7.59\left(\mathrm{~m}, 2 \mathrm{H}, \mathrm{H}_{2,4}\right), 7.06\left(\mathrm{~m}, 2 \mathrm{H}, \mathrm{H}_{1,5}\right)$, $6.87\left(\mathrm{~m}, 1 \mathrm{H}, \mathrm{H}_{10}\right), 6.71\left(\mathrm{~m}, 2 \mathrm{H}, \mathrm{H}_{8,12}\right), 2.35\left(\mathrm{~s}, 6 \mathrm{H}, \mathrm{H}_{13,14}\right)$.

${ }^{13} \mathbf{C}$ NMR $\left\{{ }^{1} \mathbf{H}\right\}\left(\mathbf{5 0} \mathbf{~ M H z}, \mathbf{C D C l}_{3}\right): \delta 160.78\left(\mathrm{C}_{6}\right), 155.65\left(\mathrm{C}_{7}\right), 140.01$ $\left(\mathrm{C}_{9,11}\right), 127.04\left(\mathrm{q},{ }^{3} \mathrm{~J}_{\mathrm{CF}}=3.8 \mathrm{~Hz}, \mathrm{C}_{2,4}\right), 126.25\left(\mathrm{C}_{10}\right), 124.59\left(\mathrm{q},{ }^{2} \mathrm{~J}_{\mathrm{CF}}=32.7\right.$ $\left.\mathrm{Hz}, \mathrm{C}_{3}\right), 118.92\left(\mathrm{q},{ }^{1} \mathrm{~J}_{\mathrm{CF}}=271.1 \mathrm{~Hz}, \mathrm{C}_{15}\right), 117.78\left(\mathrm{C}_{8,12}\right), 117.63\left(\mathrm{C}_{1,5}\right)$, $21.26\left(\mathrm{C}_{13,14}\right)$.

${ }^{19}$ F NMR $\left\{{ }^{1} \mathbf{H}\right\}\left(235 \mathrm{MHz}, \mathrm{CDCl}_{3}\right): \delta-62.11$ (s).

Elemental analysis: Calculated for $\mathrm{C}_{15} \mathrm{H}_{13} \mathrm{~F}_{3} \mathrm{O}: \mathrm{C}, 67.66 ; \mathrm{H}, 4.92$; F, 21.41. Found: C, 67.37; H, 5.03; F, 21.80.

GC/MS (EI): $\mathrm{rt}=16.71 \mathrm{~min}, \mathrm{M} / \mathrm{Z}=266$.

IR $\left(\mathbf{C H}_{\mathbf{2}} \mathbf{C l}_{\mathbf{2}}\right): v\left(\mathrm{~cm}^{-1}\right)=3053,2985,1615,1591,1513,1326\left(\mathrm{CF}_{3}\right), 1237$ (C-O), $1169\left(\mathrm{CF}_{3}\right), 1123,1066,840$.

$\mathbf{R}_{\mathbf{f}}: 0.68$ (eluent: hexanes). 
9) 3',5'-dimethyl-4-methoxy-diphenylether

Experimental procedure (Table 5, entry 14)

Following the general procedure $\left(82{ }^{\circ} \mathrm{C}, 48\right.$ hours $), 3,5$-dimethylphenol $(244 \mathrm{mg}, 2.0 \mathrm{mmol})$ was coupled with 4-iodoanisole $(655 \mathrm{mg}, 2.8 \mathrm{mmol})$ using cesium carbonate $(1.303 \mathrm{~g}, 4.0 \mathrm{mmol}), \mathrm{Cu}_{2} \mathrm{O}(14.4 \mathrm{mg}$, $0.1 \mathrm{mmol})$, Chxn-Py-Al (117 mg, $0.4 \mathrm{mmol})$, activated and powdered $3 \AA$ molecular sieves $(600 \mathrm{mg})$, and acetonitrile $(1.12 \mathrm{~mL})$. The crude product was dried for 2 hours at $100{ }^{\circ} \mathrm{C}$ and then purified by flash chromatography on silica gel (eluent: hexanes) to provide $420 \mathrm{mg}$ (92\% yield) of the desired product as a colorless solid which can be recrystallized in petroleum ether.

\section{Identification}

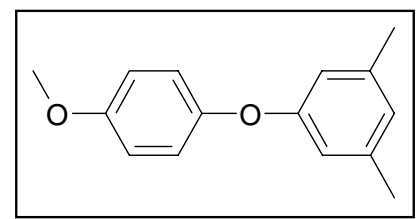

Mp: $67^{\circ} \mathrm{C}$ (petroleum ether) (Lit. ${ }^{12}: 67^{\circ} \mathrm{C}$ ).

${ }^{1} \mathbf{H}$ NMR $\left(\mathbf{2 0 0} \mathbf{~ M H z}, \mathbf{C D C l}_{\mathbf{3}}\right): \delta 6.99-7.06(\mathrm{~m}, 2 \mathrm{H}), 6.88-6.99(\mathrm{~m}, 2 \mathrm{H}), 6.74$ $(\mathrm{m}, 1 \mathrm{H}), 6.64(\mathrm{~m}, 2 \mathrm{H}), 3.85$ (s, 3H, MeO), $2.32(\mathrm{~s}, 6 \mathrm{H}, \mathrm{Me})$.

${ }^{13} \mathbf{C}$ NMR $\left\{{ }^{1} \mathbf{H}\right\}\left(\mathbf{5 0} \mathbf{~ M H z}, \mathbf{C D C l}_{3}\right): \delta 158.52(\mathrm{Cq}), 155.76(\mathrm{Cq}), 150.26(\mathrm{Cq})$, $139.45(2 \mathrm{Cq}), 124.22(\mathrm{CH}), 120.84(2 \mathrm{CH}), 115.31(2 \mathrm{CH}), 114.77(2 \mathrm{CH})$, $55.59\left(\mathrm{CH}_{3}\right), 21.35\left(2 \mathrm{CH}_{3}\right)$.

GC/MS (EI): $\mathrm{rt}=19.77 \mathrm{~min}, \mathrm{M} / \mathrm{Z}=228$.

$\mathbf{R}_{\mathbf{f}}: 0.61$ (eluent: hexanes).

10) 2-(3,5-dimethyl-phenoxy)-pyridine

Experimental procedure (Table 5, entry 15)

Following the general procedure $\left(110{ }^{\circ} \mathrm{C}, 24\right.$ hours $), 3,5$-dimethylphenol $(244 \mathrm{mg}, 2.0 \mathrm{mmol})$ was coupled with 2-bromopyridine $(292 \mu \mathrm{L}, 3.0 \mathrm{mmol})$ using cesium carbonate $(1.303 \mathrm{~g}, 4.0 \mathrm{mmol}), \mathrm{Cu}_{2} \mathrm{O}(14.4 \mathrm{mg}$, $0.1 \mathrm{mmol})$, Chxn-Py-Al (117 mg, $0.4 \mathrm{mmol})$, activated and powdered $3 \AA$ molecular sieves $(600 \mathrm{mg})$, and acetonitrile $(1.2 \mathrm{~mL})$. The crude oily residue was dried for 2 hours at $100{ }^{\circ} \mathrm{C}$ and then purified by flash chromatography on silica gel (eluent: gradient hexanes / dichloromethane 100/0 to 85/15) to provide $371 \mathrm{mg}$ (93 $\%$ yield) of the desired product as a yellow oil. This compound is not kown in literature.

\section{$\underline{\text { Identification }}$}

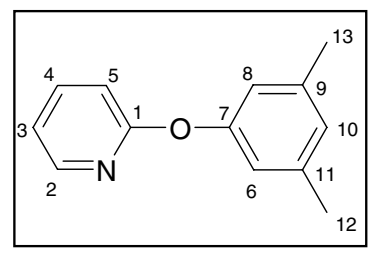

${ }^{1} \mathbf{H}$ NMR $\left(250 \mathbf{~ M H z}, \mathbf{C D C l}_{\mathbf{3}}\right): \delta 8.21\left(\mathrm{ddd}, 1 \mathrm{H},{ }^{3} \mathrm{~J}_{\mathrm{HH}}=5.0 \mathrm{~Hz},{ }^{4} \mathrm{~J}_{\mathrm{HH}}=2.0 \mathrm{~Hz}\right.$, $\left.{ }^{5} \mathrm{~J}_{\mathrm{HH}}=0.7 \mathrm{~Hz}, \mathrm{H}_{2}\right), 7.66\left(\mathrm{ddd}, 1 \mathrm{H},{ }^{3} \mathrm{~J}_{\mathrm{HH}}=8.2 \mathrm{~Hz},{ }^{3} \mathrm{~J}_{\mathrm{HH}}=7.2 \mathrm{~Hz},{ }^{4} \mathrm{~J}_{\mathrm{HH}}=2.0\right.$ $\left.\mathrm{Hz}, \mathrm{H}_{4}\right), 6.97\left(\mathrm{ddd}, 1 \mathrm{H},{ }^{3} \mathrm{~J}_{\mathrm{HH}}=7.2 \mathrm{~Hz},{ }^{3} \mathrm{~J}_{\mathrm{HH}}=5.0 \mathrm{~Hz},{ }^{4} \mathrm{~J}_{\mathrm{HH}}=0.9 \mathrm{~Hz}, \mathrm{H}_{3}\right)$, $6.88\left(\mathrm{ddd}, 1 \mathrm{H},{ }^{3} \mathrm{~J}_{\mathrm{HH}}=8.2 \mathrm{~Hz},{ }^{4} \mathrm{~J}_{\mathrm{HH}}=0.9 \mathrm{~Hz},{ }^{5} \mathrm{~J}_{\mathrm{HH}}=0.7 \mathrm{~Hz}, \mathrm{H}_{5}\right), 6.84(\mathrm{~m}, 1 \mathrm{H}$, $\left.\mathrm{H}_{10}\right), 6.76\left(\mathrm{~m}, 2 \mathrm{H}, \mathrm{H}_{6,8}\right), 2.32$ (s, 6H, $\left.\mathrm{H}_{12,13}\right)$.

${ }^{13} \mathbf{C}$ NMR $\left\{{ }^{1} \mathbf{H}\right\}\left(\mathbf{5 0} \mathbf{~ M H z}, \mathbf{C D C l}_{3}\right): \delta 164.02\left(\mathrm{C}_{1}\right), 154.15\left(\mathrm{C}_{7}\right), 147.87\left(\mathrm{C}_{2}\right)$, $139.47\left(\mathrm{C}_{9,11}\right), 139.27\left(\mathrm{C}_{4}\right), 126.53\left(\mathrm{C}_{10}\right), 118.80\left(\mathrm{C}_{6,8}\right), 118.22\left(\mathrm{C}_{5}\right), 111.47$ $\left(\mathrm{C}_{3}\right), 21.34\left(\mathrm{C}_{12,13}\right)$.

Elemental analysis: Calculated for $\mathrm{C}_{13} \mathrm{H}_{13} \mathrm{NO}$ : C, 78.21; H, 6.69; N, 7.04. Found: C, 78.36; H, 6.58; N, 7.03.

GC/MS (EI): $\mathrm{rt}=17.65 \mathrm{~min}, \mathrm{M} / \mathrm{Z}=199$.

IR $(\mathbf{K B r}): v\left(\mathrm{~cm}^{-1}\right)=3057,3051,3013,2918,2856,1616,1583,1572$, 1467, 1429, 1296, 1246 (C-O), 1138, 856, 779.

$\mathbf{R}_{\mathbf{f}}$ : 0.22 (eluent: hexanes / dichloromethane 75/25).

11) 2,3',5'-trimethyl-diphenylether

Experimental procedure (Table 5, entry 16)

Following the general procedure $\left(82{ }^{\circ} \mathrm{C}, 118\right.$ hours $), 3,5$-dimethylphenol $(244 \mathrm{mg}, 2.0 \mathrm{mmol})$ was coupled with 2-iodotoluene $(383 \mu \mathrm{L}, 3.0 \mathrm{mmol})$ using cesium carbonate $(1.303 \mathrm{~g}, 4.0 \mathrm{mmol}), \mathrm{Cu}_{2} \mathrm{O}(14.4 \mathrm{mg}$, 
$0.1 \mathrm{mmol})$, Chxn-Py-Al (117 mg, $0.4 \mathrm{mmol})$, activated and powdered $3 \AA$ molecular sieves $(600 \mathrm{mg})$, and acetonitrile $(1.2 \mathrm{~mL})$. The crude oily residue was purified by flash chromatography on silica gel (eluent: hexanes) to provide $399 \mathrm{mg}$ ( $94 \%$ yield) of the desired product as an orange oil.

\section{$\underline{\text { Identification }}$}

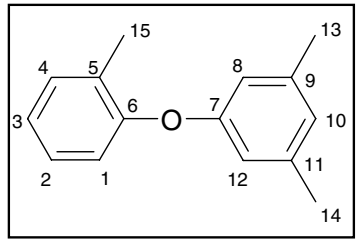

${ }^{1}$ H NMR (200 MHz, CDCl $): \delta^{13} 7.08-7.33(\mathrm{~m}, 3 \mathrm{H}), 6.95-6.99(\mathrm{~m}, 1 \mathrm{H})$, $6.76(\mathrm{~m}, 1 \mathrm{H}), 6.61\left(\mathrm{~m}, 2 \mathrm{H}, \mathrm{H}_{8,12}\right), 2.33$ (s, 6H, H $\left.\mathrm{H}_{13,14}\right), 2.32$ (s, 3H, H $\mathrm{H}_{15}$ ).

${ }^{13} \mathbf{C} \mathbf{N M R}\left\{{ }^{1} \mathbf{H}\right\}\left(\mathbf{5 0} \mathbf{~ M H z}, \mathbf{C D C l}_{3}\right): \delta^{13} 157.94(\mathrm{Cq}), 154.69(\mathrm{Cq}), 139.55$

$\left(\mathrm{C}_{9,11}\right), 131.41(\mathrm{CH}), 130.02\left(\mathrm{C}_{5}\right), 127.14(\mathrm{CH}), 124.22(\mathrm{CH}), 123.83(\mathrm{CH})$, $119.81(\mathrm{CH}), 115.11\left(\mathrm{C}_{8,12}\right), 21.42\left(\mathrm{C}_{13,14}\right), 16.30\left(\mathrm{C}_{15}\right)$.

GC/MS (EI): $\mathrm{rt}=17.46 \mathrm{~min}, \mathrm{M} / \mathrm{Z}=212$.

$\mathbf{R}_{\mathbf{f}}: 0.26$ (eluent: hexanes).

\section{2) $2,2^{\prime}$-dimethyl-diphenylether ${ }^{14}$}

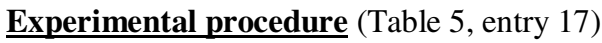

Following the general procedure $\left(110{ }^{\circ} \mathrm{C}, 35\right.$ hours $), o$-cresol $(206 \mu \mathrm{L}, 2.0 \mathrm{mmol})$ was coupled with 2iodotoluene $(383 \mu \mathrm{L}, 3.0 \mathrm{mmol})$ using cesium carbonate $(1.303 \mathrm{~g}, 4.0 \mathrm{mmol}), \mathrm{Cu}_{2} \mathrm{O}(14.4 \mathrm{mg}, 0.1 \mathrm{mmol}), \mathrm{Chxn}-$ Py-Al (117 mg, $0.4 \mathrm{mmol})$, activated and powdered $3 \AA$ molecular sieves (600 mg), and DMF (1.2 mL). The crude oily residue was purified by flash chromatography on silica gel (eluent: hexanes) to provide $389 \mathrm{mg}$ (98\% yield) of the desired product as a colorless oil.

\section{$\underline{\text { Identification }}$}

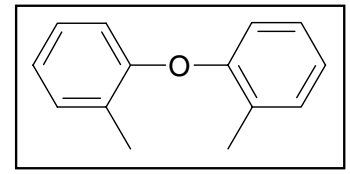

${ }^{1} \mathbf{H}$ NMR (200 MHz, $\left.\mathbf{C D C l}_{3}\right): \delta 7.32(\mathrm{~m}, 2 \mathrm{H}), 7.04-7.25(\mathrm{~m}, 4 \mathrm{H}), 6.81(\mathrm{~m}$, $2 \mathrm{H}), 2.38\left(\mathrm{~s}, 6 \mathrm{H}, \mathrm{CH}_{3}\right)$.

${ }^{13} \mathbf{C}$ NMR $\left\{{ }^{1} \mathbf{H}\right\}\left(\mathbf{5 0} \mathbf{~ M H z}, \mathbf{C D C l}_{3}\right): \delta 155.35(\mathrm{Cq}), 131.39(\mathrm{CH}), 128.90$ $(\mathrm{Cq}), 127.09(\mathrm{CH}), 123.11(\mathrm{CH}), 117.74(\mathrm{CH}), 16.25\left(\mathrm{CH}_{3}\right)$.

GC/MS (EI): $\mathrm{rt}=16.10 \mathrm{~min}, \mathrm{M} / \mathrm{Z}=198$.

$\mathbf{R}_{\mathbf{f}}: 0.40$ (eluent: hexanes).

\section{3) 4-nitro-diphenylether}

\section{Experimental procedure (Table 5, entry 18)}

Following the general procedure $\left(82^{\circ} \mathrm{C}, 24\right.$ hours $)$, phenol $(188 \mathrm{mg}, 2.0 \mathrm{mmol})$ was coupled with 4 iodonitrobenzene $(747 \mathrm{mg}, 3.0 \mathrm{mmol})$ using cesium carbonate $(1.303 \mathrm{~g}, 4.0 \mathrm{mmol}), \mathrm{Cu}_{2} \mathrm{O}(14.4 \mathrm{mg}, 0.1 \mathrm{mmol})$, Chxn-Py-Al (117 mg, $0.4 \mathrm{mmol})$, activated and powdered $3 \AA$ molecular sieves $(600 \mathrm{mg})$, and acetonitrile (1.2 $\mathrm{mL}$ ). The crude product was purified by flash chromatography on silica gel (eluent: hexanes) to provide $400 \mathrm{mg}$ (93\% yield) of the desired product as a light yellow solid, which can be recrystallized in petroleum ether / diethylether.

\section{$\underline{\text { Identification }}$}

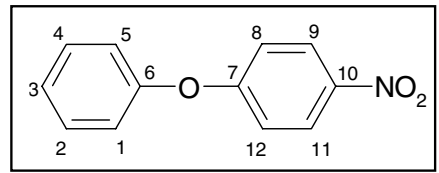

Mp: $60{ }^{\circ} \mathrm{C}$ (petroleum ether / diethylether) $\left(\right.$ Lit. $\left.^{15}: 60{ }^{\circ} \mathrm{C}, \mathrm{MeOH}\right)$.

${ }^{1} \mathbf{H}$ NMR (200 MHz, CDCl 3 ): $\delta$ 8.15-8.21 (m, 2H, H, $\left.{ }_{9,11}\right), 7.40-7.48(\mathrm{~m}, 2 \mathrm{H}$, $\left.\mathrm{H}_{2,4}\right)$, 7.21-7.30 (m, $\left.1 \mathrm{H}, \mathrm{H}_{3}\right), 7.07-7.13\left(\mathrm{~m}, 2 \mathrm{H}, \mathrm{H}_{1,5}\right), 6.98-7.05(\mathrm{~m}, 2 \mathrm{H}$, $\mathrm{H}_{8,12}$.

${ }^{13} \mathbf{C}$ NMR $\left\{{ }^{1} \mathbf{H}\right\}\left(\mathbf{5 0} \mathbf{~ M H z}, \mathbf{C D C l}_{3}\right): \delta 163.38\left(\mathrm{C}_{7}\right), 154.72\left(\mathrm{C}_{6}\right), 142.64\left(\mathrm{C}_{10}\right)$, $130.34\left(\mathrm{C}_{2,4}\right), 125.94\left(\mathrm{C}_{9,11}\right), 125.44\left(\mathrm{C}_{3}\right), 120.55\left(\mathrm{C}_{1,5}\right), 117.10\left(\mathrm{C}_{8,12}\right)$.

GC/MS (EI): $\mathrm{rt}=19.99 \mathrm{~min}, \mathrm{M} / \mathrm{Z}=215$.

$\mathbf{R}_{\mathbf{f}}: 0.37$ (eluent: hexanes). 
A General and Mild Ullmann-Type Synthesis of Diaryl Ethers

${ }^{1}$ H NMR spectrum of 3',5'-dimethyl-4-trifluoromethyldiphenylether (Table 5, Entry 13). $\left(200 \mathrm{MHz}, \mathrm{CDCl}_{3}\right)$ : 
A General and Mild Ullmann-Type Synthesis of Diaryl Ethers

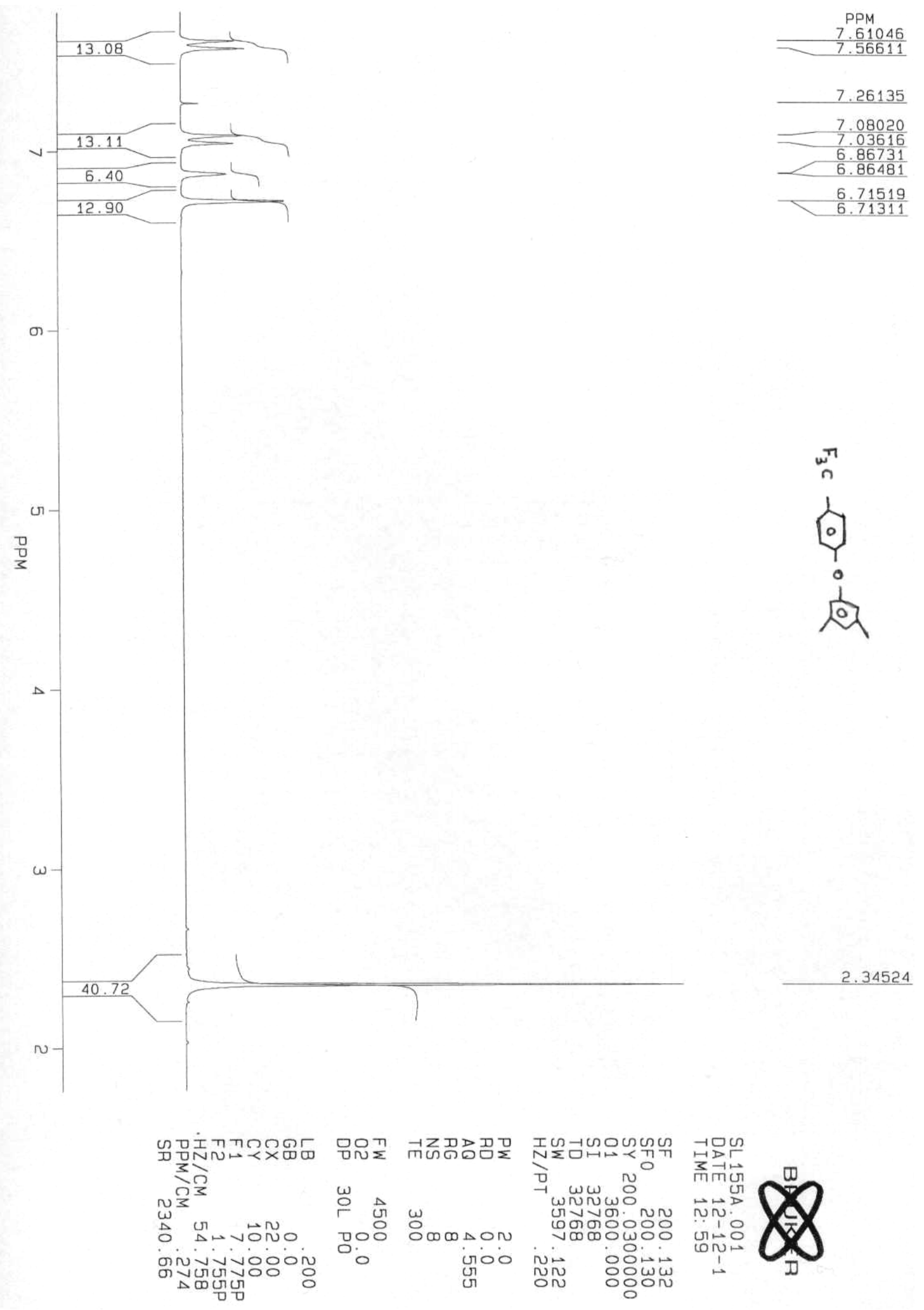

\section{Reference Section:}


${ }^{1}$ Burfield, D. R. J. Org. Chem. 1977, 42, 3060-3065.

2 Dieter, R. K.; Silks III, L. A.; Fishpaugh, J. R.; Kastner, M. E. J. Am. Chem. Soc. 1985, 107, 4679-4692.

${ }^{3}$ Perrin, D. D.; Armarego, W. L. F.; Perrin, D. R. Purification of Laboratory Chemicals, 3rd ed.; Pergamon Press: New-York, 1985.

${ }^{4}$ Belokon, Y. N.; North, M.; Churkina, T. D.; Ikonnikov, N. S.; Maleev, V. I. Tetrahedron 2001, 57, 2491-2498.

${ }^{5}$ Ullmann, F.; Sponagel, P. Justus Liebig's Ann. Chem. 1906, 350, 83-107.

${ }^{6}$ Byers, C. H.; Williams, D. F. J. Chem. Eng. Data 1987, 32, 344-348.

${ }^{7}$ Harvey, L.; Gleicher, G. J.; Totherow, W. D. Tetrahedron 1969, 25, 5019-5026.

${ }^{8}$ Haga, N.; Takayanagi, H. J. Org. Chem. 1996, 61, 735-745.

${ }^{9}$ Nevalainen, T.; Kolehmainen, E.; Magn. Reson. Chem. 1994, 32, 480-486.

${ }^{10}$ Aranyos, A.; Old, D. W.; Kiyomori, A.; Wolfe, J. P.; Sadighi, J. P.; Buchwald, S. L. J. Am. Chem. Soc. 1999, 121, 4369-4378.

${ }^{11}$ Mine, A.; Kamoshita, K.; Hino, N.; Nakai, S. DE Patent 1912600, 1969; Chem. Abstr. 1970, 72, 21511.

${ }^{12}$ Walter Barell-Festschr.; Basel 1936, 266-273.

${ }^{13}$ Marcoux, J.-F.; Doye, S.; Buchwald, S. L. J. Am. Chem. Soc. 1997, 119, 10539-10540, Supporting Information.

${ }^{14}$ Voegtle, F.; Kleiner, T.; Leppkes, R.; Laeubli, M. W.; Ammann, D.; Simon, W. Chem. Ber. 1983, 116, $2028-$ 2034.

${ }^{15}$ Forrest, J. J. Chem. Soc. 1960, 581-588. 